Methods of polarographic
analysis

\section{Emil Paleček}

Polarography of Molecules of Biological Significance. Edited by W. Franklin Smyth. Pp.328. (Academic: London and New York, 1979.) £22.60, \$52.

THE sensitivity of polarographic (voltammetric) determinations has greatly increased in the past $10-15$ years. While the limits of detection of organic and inorganic substances reached by classical d.c. polarographic methods were usually around $10^{-5} \mathrm{M}$, using modern differential pulse anodic stripping voltammetry, inorganic substances can be determined at concentrations down to $10^{-10} \mathrm{M}$. Cathodic stripping voltammetry is becoming a very useful tool in the analysis of certain organic compounds at concentrations as low as $10^{-8}-10^{-9} \mathrm{M}$; and differential (derivative) pulse polarography operates at concentrations of $10^{-6}-10^{-8} \mathrm{M}$ for both organic and inorganic substances. This remarkable gain in sensitivity, together with the commercial availability of reliable polarographic analysers, has resulted in a steady increase in the number of analysts using modern polarographic (voltammetric) methods. This process, sometimes called the 'renaissance of polarography', is, however, not yet accompanied by the predictable abundance of books concerning the theory and application of modern polarographic methods. Smyth's book is thus a valuable acquisition for analytical chemistry libraries.

The first two chapters of this book describe unit processes in polarographic (voltammetric) analysis of organic substances such as choice of method, preparation of the sample, selection of a proper supporting electrolyte, cell and electrodes, derivatization and complexation procedures, and so on. Further chapters deal with the application of polarographic methods in pharmacy and pharmacology, basic medical and environmental sciences, and agriculture. Polarographic determinations of various groups of substances are in general compared with determinations by means of other methods; and the advantages of the polarographic analysis of certain types of compounds are stressed. It is shown that differential pulse polarography is highly suitable for the determination of pharmaceutical products and other substances containing nitrogroups; and sulphur-containing substances (many of them representing trace foreign materials which pollute the environment) can be determined at low concentrations by cathodic stripping voltammetry.

The book is well-written; it has not, however, managed to escape entirely without errors; for example, in the paragraph on purine and its derivatives (Chapter 5) it is stated that pyrimidine and cytosine are oxidizable at graphite and mercury electrodes. It follows from the cited paper (G. Dryhurst and P. J. Elving) that both substances are non-oxidizable. In spite of the fact that a relatively extensive literature on polarography of nucleic acids exists, only two original papers are cited in Chapter 6, one of them incorrectly; review articles concerning this topic are

\section{Interstellar dust}

\section{D.E. Brownlee}

Cosmic Dust: Its Impact on Astronomy. By Peter G. Martin. Pp.266. (Clarendon/Oxford University Press: Oxford, 1979.) $£ 9$.

Peter MARTIN's Cosmic Dust: Its Impact on Astronomy is not an encyclopaedia of what is known about extraterrestrial dust. It does not cover Moon dust, Mars dust, comet dust, Venusian smog or plumes from Io's volcanoes. It is a book which concentrates on star dust and is a comprehensive coverage of current information and ideas concerning dust in the interstellar medium. Interstellar dust is a basic form of matter in galaxies and is the common form of most elements heavier than helium that are not currently residing in stars. Interstellar grains are produced in gaseous regions surrounding stars; they experience an evolutionary existence as tiny astronomical bodies and are eventually destroyed by super novae shocks or interactions with stars. They are somewhat of a nuisance, as they totally obscure most of our Galaxy in visual wavelengths and cause extinction, reddening and polarization in observable objects. However, as Dr Martin enthusiastically points out, dust grains are fascinating bodies important to a variety of disciplines in astronomy.

The book's first five chapters deal with the interaction of dust and radiation in the interstellar medium. These chapters cover fundamental theory as well as up-to-date observational and laboratory data. Topics include radiation transfer, calculation and laboratory measurement of particle crosssections, extinction of optical and X-ray wavelengths, polarization, and scattering. The fifth chapter deals with the properties and modelling of reflection nebulae. In addition to providing the reader with a good background on the interaction of light and dust, the author also interprets existing observations in terms of limitations they place on the nature and distribution of interstellar dust.

Chapters six and seven discuss the theory and observation of thermal emission from grains. This includes far-infrared from the

\section{completely ignored.}

Minor errors do not, however, substantially diminish the significance of this book, which can be confidently recommended to all analytical chemists dealing with determinations of biologically important organic substances, and especially to those of them who have come in contact with polarography in the past. $\square$

Emil Palecek is Head of the Department of Macromolecular Biophysics at the Institute of Biophysics, Czechoslovak Academy of Sciences, Brno, Czechoslovakia.

Galactic plane and the properties and structure of circumstellar dust shells. Chapters eight and nine deal with the distribution of dust in the Galaxy, the nature of dust clouds and interactions of dust with the interstellar environment. Specific topics include radiation pressure, spin, charge, sputtering, molecule formation, alignment and dust in dark clouds, and $\mathrm{H}$ II regions, planetary nebulae and the Solar System. The coverage of most of these topics is somewhat introductory and does not go into considerable depth. In chapter ten the significance of spectral signatures such as the $2200 \AA$ and $10 \mu \mathrm{m}$ features are discussed as indicators of grain composition. Also discussed are the importance of cosmic abundances and abundances in interstellar gas as constraints on grain composition. Chapter eleven reviews the evolutionary processes and timescales experienced by dust. Basically this chapter is concerned with the formation of refractory cores and volatile mantles and their stability in changing interstellar environments. The final chapter contains a brief section on dust in other galaxies and an intriguing discussion about properties and possible observations of dust between galaxies.

In the preface the author states a goal to give the reader basic tools and to discuss concepts underlying current research. This he does quite well. A careful reading of this book will provide the reader with a broadbased background in both the theory and current state of observation of interstellar grains. In general the book can be highly recommended, although there are some minor drawbacks. The organization is such that certain topics are diffused through widely separated parts of the book. It sometimes is difficult to answer a specific question without reading most of the book. Although it provides a good background, this is not a handy source book for quick reference. The most serious drawback in the opinion of this reviewer is the lack of formal references. At the end of each chapter is a list of "further reading" but these are not true references that refer to specific points in the text. This continually raises a question of credibility. The reader is apparently expected to accept the written word as dogma. The lack of conventional references also makes it difficult for the 